\title{
Etude de la variabilité individuelle chez le maïs à l'issue de la phase d'implantation - conséquences sur la croissance et la production de grain
}

\author{
B. Pommel ${ }^{1}$ et A. Fleury ${ }^{2}$ \\ 1 INRA, Laboratoire d'Agronomie. F-78850 Thiverval-Grignon, France; \\ 2 Chaire d'Agronomie. Institut National Agronomique Paris-Grignon, 16, rue Claude-Bernard F-75231 Paris Cedex 05, France
}

(reçu le 10 juin 1988, accepté le 13 février 1989)

Résumé - La croissance et le développement d'environ 400 individus, déterminés préalablement au hasard au sein d'un même peuplement de maïs au champ, ont été analysés de la levée à la récolte. La faible densité de semis limite la compétition.

La levée est étalée sur 9 jours, les plantes précoces ou tardives étant de façon préférentielle localisées à proximité de plantes du même type. Les manques à la levée se retrouvent plus souvent environnés de plantes précoces. Les plantes tardives ont généralement un comportement normal quand elles sont situées en zone tardive, alors qu'elles sont pénalisées quand elles sont situées en zone précoce.

Parmi les plantes levées le même jour, on observe des différences de croissance, que l'on peut rapporter à leur poids à l'origine: elles reflètent, sans doute, une variabilité existant au sein du lot de semence.

Les origines de la structure observée au sein du peuplement postérieurement à la levée et l'évolution ultérieure de cette structure jusqu'au rendement individuel en grain sont discutées.

Zea mays - levée - implantation - hétérogénéité - taux de croissance

Summary - Plant to plant variability among maize seedlings : consequences on growth and grain yield. Growth and development of about 400 plants randomly fixed within an experimental plot have been analyzed from emergence to grain harvest. The low sowing density limits competition.

Germination was spread out over 9 days. The precocious or late plants are more often located near plants of the same type. Areas lacking in germination were more frequently found surrounded by precocious plants. The late plants generally grow normally when located in a late zone, but are penalized when located in a precocious zone.

Among plants which emerged on the same day we observed differences in growth, which were dependent on their weight at the beginning and which probably express the variability within the seed lot.

We discuss the origins of the structure of the crop after emergence, and the subsequent evolution of this structure, until the individual grain yield.

Zea mays - emergence - establishment - heterogeneousness - growth rate

\section{Introduction}

Lors de l'étude du comportement d'une parcelle de maïs, la variabilité interne du peuplement est plutôt perçue comme une source de complications, considérée comme inévitable et rarement prise en compte. On peut penser avec Pendleton et Seif (1962), qu'elle n'est préjudiciable au rendement que si le gain des individus dominants est inférieur à la perte des dominés. Ce n'est pas le cas d'espèces où l'indice de récolte est stable vis-à-vis du poids individuel (colza, céréales...), mais peut l'être chez le maïs, à cause des caractères unique et défini de l'épi femelle, du moins chez les cultivars précoces. Ainsi Navarro Garza (1984) constate que le rapport du nombre de grains au poids végétatif est meilleur pour les pieds de taille moyenne que pour les extrêmes. Les travaux de Glenn et Daynard (1974), d'Edmeades et Daynard (1979) montrent une dépression modérée (inférieure à 10\%) du rendement dans différents systèmes de modélisation de l'hétérogénéité. 
Duncan et al. (1972) associent l'effet dépressif d'un facteur limitant sur le rendement à un accroissement d'hétérogénéité, c'est-à-dire à une compétition plus intense. Cependant la position des individus dans un tel rapport est surtout héritée d'une différenciation antérieure de la structure de peuplement, dont Fleury et Caneill (1983) montrent, sur betterave, qu'elle est largement créée par l'échelonnement de la levée et la variabilité des états très précoces de croissance. Cette dernière dépend de la qualité du lot de semence (Abdul Hamid, 1986), de la dispersion des poids des graines (Abd el Rahman et Bourdu, 1986) et, probablement, des conditions de la phase germination-levée (Belfqih et Charbey (1984) et Maillet (1987) sur la betterave).

Le déroulement de la phase juvénile peut donc engendrer deux types de conséquences sur le peuplement en modifiant d'une part la date du début de fonctionnement photosynthétique, d'autre part la vitesse de croissance. C'est ce que nous avons étudié chez le maīs sur un peuplement peu dense, choisi en sorte qu'il n'y ait pas de compétition engendrant une amplification de l'effet originel attendu. C'est pourquoi un hybride simple très précoce, de format réduit, a été retenu, cultivé à faible densité. Deux groupes d'indicateurs ont été saisis à l'échelle individuelle :

- paramètres de croissance en cours de végétation;
- état de croissance et production jugés à maturité de toutes les plantes.

Le souci de décrire à l'échelle individuelle a impliqué un suivi non destructif de la croissance, évalué par des méthodes corrélatives.

En travaillant au champ, nous avons voulu, en l'absence de références, nous situer dans l'ordre de grandeur d'effets qui pourraient être observés en conditions agricoles.

\section{Matériel et Méthodes}

\section{Conditions de culture}

La parcelle étudiée fait partie du champ expérimental de Grignon (Yvelines). Le sol est un limon argileux profond, bien pourvu en matière organique, dont les teneurs en phosphore (300 ppm Joret Hébert) et en potassium échangeable (1000 méq pour 100 grammes de terre) ont paru suffisantes pour une alimentation correcte (Pommel, 1979), ainsi que l'apport azoté (150 $\mathrm{kg}$ à l'hectare). Le climat a été favorable (Tableau I) avec des températures moyennes, un assez bon ensoleillement et des précipitations un peu faibles pour la localité, mais bien situées juste après floraison.

Le cultivar précoce Borée LG1 a été semé à 60000 grains/ha au moyen d'un semoir à distribution mécanique sur 2 rangs écartés de $85 \mathrm{~cm}$. Afin d'assurer l'étalement de la levée, on a limité l'affinement du lit de semences. L'humidité de la terre au voisinage des semences, assez variable (moyenne : 19\%; écarttype : 2,9 sur 19 échantillons), laisse penser qu'un certain nombre de graines ont eu une imbibition lente,

Tableau I. Données climatologiques de la campagne 1985, comparativement aux moyennes locales établies sur 21 ans.

\begin{tabular}{|c|c|c|c|c|c|c|c|}
\hline Mois & Décade & $\begin{array}{l}\text { Tempér. } \\
1985\end{array}$ & $\begin{array}{l}\text { Tempér. } \\
\text { moyenne }\end{array}$ & $\begin{array}{l}\text { Précip. } \\
1985\end{array}$ & $\begin{array}{l}\text { Précip. } \\
\text { moyenne }\end{array}$ & $\begin{array}{l}\text { Insola. } \\
1985\end{array}$ & $\begin{array}{l}\text { Insola. } \\
\text { moyenne }\end{array}$ \\
\hline Avril & $\begin{array}{l}2 \\
3\end{array}$ & $\begin{array}{r}10,0 \\
7,7\end{array}$ & $\begin{array}{l}9,2 \\
9,7\end{array}$ & $\begin{array}{r}14,9 \\
7,1\end{array}$ & $\begin{array}{l}12,5 \\
14,0\end{array}$ & & $\begin{array}{l}5,6 \\
5,6\end{array}$ \\
\hline Mai & $\begin{array}{l}1 \\
2 \\
3\end{array}$ & $\begin{array}{r}9,9 \\
13,3 \\
15,2\end{array}$ & $\begin{array}{l}11,1 \\
13,0 \\
13,1\end{array}$ & $\begin{array}{r}8,8 \\
46,8 \\
5,8\end{array}$ & $\begin{array}{l}21,9 \\
19,7 \\
24,8\end{array}$ & $\begin{array}{l}2,2 \\
4,0 \\
8,5\end{array}$ & $\begin{array}{l}5,1 \\
6,2 \\
5,3\end{array}$ \\
\hline Juin & $\begin{array}{l}1 \\
2 \\
3\end{array}$ & $\begin{array}{l}16,7 \\
13,3 \\
14,0\end{array}$ & $\begin{array}{l}15,1 \\
15,7 \\
15,9\end{array}$ & $\begin{array}{r}20,2 \\
1,4 \\
20,2\end{array}$ & $\begin{array}{l}18,1 \\
14,1 \\
21,2\end{array}$ & $\begin{array}{l}8,9 \\
6,3 \\
3,4\end{array}$ & $\begin{array}{l}7,2 \\
6,6 \\
6,2\end{array}$ \\
\hline Juillet & $\begin{array}{l}1 \\
2 \\
3\end{array}$ & $\begin{array}{l}17,9 \\
18,0 \\
18,4\end{array}$ & $\begin{array}{r}17,9 \\
18,0 \\
18,3\end{array}$ & $\begin{array}{r}0,4 \\
17,9 \\
31,4\end{array}$ & $\begin{array}{l}17,3 \\
26,1 \\
19,4\end{array}$ & $\begin{array}{r}10,7 \\
9,0 \\
6,8\end{array}$ & $\begin{array}{l}7,4 \\
6,3 \\
7,2\end{array}$ \\
\hline Août & $\begin{array}{l}1 \\
2 \\
3\end{array}$ & $\begin{array}{l}15,2 \\
16,2 \\
16,6\end{array}$ & $\begin{array}{r}18,3 \\
17,8 \\
16,9\end{array}$ & $\begin{array}{l}12,1 \\
12,3 \\
11,0\end{array}$ & $\begin{array}{l}24,6 \\
15,6 \\
12,5\end{array}$ & $\begin{array}{l}6,3 \\
6,1 \\
8,8\end{array}$ & $\begin{array}{l}6,3 \\
6,5 \\
6,6\end{array}$ \\
\hline Septembre & $\begin{array}{l}1 \\
2 \\
3\end{array}$ & $\begin{array}{l}14,1 \\
16,0 \\
17,6\end{array}$ & $\begin{array}{l}15,9 \\
15,0 \\
14,1\end{array}$ & $\begin{array}{r}17,3 \\
9,2 \\
0,0\end{array}$ & $\begin{array}{l}15,4 \\
20,4 \\
21,0\end{array}$ & $\begin{array}{l}8,1 \\
7,4 \\
8,6\end{array}$ & $\begin{array}{l}5,8 \\
5,5 \\
5,3\end{array}$ \\
\hline Octobre & 1 & 16,4 & 12,8 & 8,2 & 18,0 & 7,0 & 4,4 \\
\hline
\end{tabular}


d'autant plus qu'il a peu plu après le semis $(1,9 \mathrm{~mm}$ dans les 5 jours suivants, 6,3 dans les 10 jours).

\section{Protocole de mesure}

Sur 18 lignes contiguës, on a isolé après semis 3 segments disjoints longs de $1,50 \mathrm{~m}$, comportant 8 emplacements de graine. Chaque plante a été identifiée par la date de sa levée, suivie journellement à même heure; l'ensemble des plantes apparues en $24 \mathrm{~h}$ constitue un "groupe de levée».

Pour réaliser le suivi non destructif, on a régulièrement mesuré, prélevé, séché à l'étuve $\left(105^{\circ}, 48 \mathrm{~h}\right)$ et pesé 30 plantes choisies hors dispositif pour représenter toute la gamme de tailles alors rencontrée dans l'essai. La hauteur des plantes était appréciée en étirant chaque feuille et en retenant la valeur maximale observée.

Tant que la hauteur a été suffisante pour l'estimation convenable du poids par régression (dates 1 à 10), elle seule a été mesurée, la précision étant de plus ou moins $9 \%$. Plus tard, la qualité de l'estimation se dégradant, on a ajouté la mesure du diamètre à la base de la tige : le poids est alors évalué par l'équation de régression sur le produit (hauteur $x$ diamètre au carré); la précision est de plus ou moins $13 \%$.

On ne peut donc prétendre à une évaluation précise de la croissance de chaque individu; c'est pourquoi on a seulement considéré des regroupements d'au moins 15 à 20 pieds, sauf mention particulière.

Ont été mesurés :

- pour toutes les plantes (377 au maximum du peuplement) :

- la hauteur quotidienne pendant la période de levée (soit 8 mesures pour le groupe 1,7 pour le groupe 2...) : dates 1 à 8 ;

- la hauteur et le diamètre aux dates 9 et $10(29 / 05$ et 5/06), période correspondant approximativement aux stades 4 à 6 feuilles (soit juste après le passage à l'autotrophie) et à la date 15 (22/07) en pleine floraison;

- le poids de l'appareil végétatif et de grain, et dénombré les rangs et les grains de chaque épi.

- en outre pour $44 \%$ d'entre elles, situées sur 8 des 18 lignes de l'essai, la hauteur et le diamètre aux dates 11 à $14(11,19$ et $27 / 06$, et le $9 / 07)$.

Le traitement statistique des résultats a été effectué par la méthode de l'analyse de la variance. Les données climatiques proviennent de la station météorologique située sur place.

\section{Résultats}

\section{Définition des traitements expérimentaux : la levée}

A partir des 9 jours que dure l'essentiel de la levée (Fig. 1), on a constitué autant de groupes de plantes, numérotés 1 pour les premières levées, 2 pour celles levées le lendemain, et ainsi de suite. Leur effectif respectif est variable, mais reste suffisant; au moins en ce qui concerne les 7 premiers d'entre eux, pour tenter une analyse statistique. Compte tenu des valeurs observées dans le Réseau National d'Expérimentation

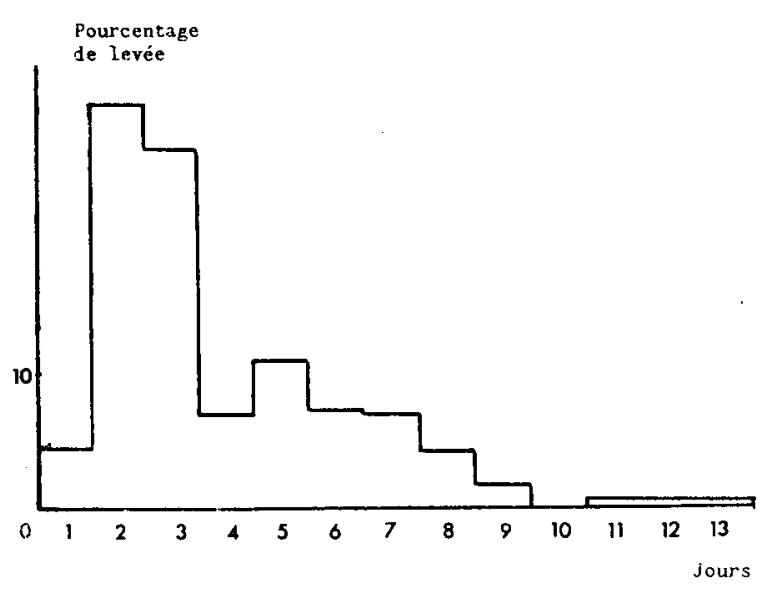

Fig. 1. Etalement de la levée.

et de Développement (RNED) Maïs (Hernandez, 1987), les valeurs moyennes de chaque composante du rendement (11,4 rangs par épi; 29 grains par rang; $356 \mathrm{mg}$ à $15 \%$ d'humidité par grain) se situent parmi les meilleures pour cette variété; le nombre de grains par rang et le poids de 1000 grains sont supérieurs aux moyennes des années 1982 à 1985. Le nombre de rangs se situe, lui, dans la moyenne générale, mais est supérieur à la moyenne de l'année 1985. Les objectifs de limiter la compétition et d'obtenir une bonne croissance ont donc été atteints. Cependant le taux de levée n'est que de $87 \%$ : la probabilité qu'une plante donnée ait 2 voisines proches (un espacement de réglage du semoir) étant de $(0,87)^{2}$, soit 0,75 , une plante sur 4 est donc en situation différente quant à la compétition. II faut donc étudier préalablement la répartition géographique des plantes et des manques à la levée.

La carte de localisation des dates de levée individuelles suggérant une répartition non aléatoire sur la parcelle, on a étudié la liaison entre les dates de levée de 2 plantes consécutives (Tableau II). Sur un segment de $\mathrm{N}$ pieds on ne connaît que $\mathrm{N}-1$ couples consécutifs; aussi le nombre de cas analysés (322) est la différence entre le nombre de pieds et le nombre de segments. On constate l'association privilégiée des levées d'une même période, précoce ou tardive, à l'exception toutefois des très tardifs qui voisinent plus souvent les précoces. Dans le but de mieux cerner la localisation des manques à la levée, on a utilisé la simplification suivante : les groupes 1 à 4 (68\% du total) ont été qualifiés de précoces $(P)$, les autres ( $32 \%$ du total) de tardifs. Sur le Tableau III, on a classé les segments :

- en fonction du nombre $\mathrm{N}$ de pieds présents,

- en fonction du nombre $n$ de pieds précoces, et comparé la fréquence observée à la fréquence théorique. 
Tableau II. Analyse de la localisation de la précocité de levée : pourcentage de pieds appartenant au groupe de levée « $j$ et suivis par un pied appartenant au groupe de levée «j». GL: Groupe de levée; THS : Significatif à 1/1000; S : Significatif à 5/100; NS : Non significatif.

\begin{tabular}{|c|c|c|c|c|c|c|c|c|c|c|}
\hline \multirow{2}{*}{$\begin{array}{l}G L \\
\text { pied }\end{array}$} & \multicolumn{2}{|c|}{ GL pied s uivant } & \multirow[b]{2}{*}{3} & \multirow[b]{2}{*}{4} & \multirow[b]{2}{*}{5} & \multirow[b]{2}{*}{6} & \multirow[b]{2}{*}{7} & \multirow[b]{2}{*}{$>8$} & \multirow[t]{2}{*}{$N b}$. & \multirow[t]{2}{*}{$\chi^{2}$} \\
\hline & 1 & 2 & & & & & & & & \\
\hline 1 & 7 & 57 & 21 & 14 & 0 & 0 & 0 & 0 & 14 & $\mathrm{~S}$ \\
\hline 2 & 6 & 46 & 31 & 3 & 7 & 1 & 2 & 3 & 95 & THS \\
\hline 3 & 8 & 20 & 40 & 8 & 12 & 1 & 4 & 7 & 92 & THS \\
\hline 4 & 5 & 37 & 32 & 16 & 11 & 0 & 0 & 5 & 19 & NS \\
\hline 5 & 0 & 23 & 29 & 10 & 10 & 16 & 13 & 0 & 31 & THS \\
\hline 6 & 0 & 4 & 13 & 0 & 17 & 21 & 29 & 17 & 24 & THS \\
\hline 7 & 0 & 8 & 8 & 4 & 8 & 38 & 13 & 21 & 24 & THS \\
\hline$>8$ & 0 & 23 & 23 & 5 & 5 & 14 & 27 & 0 & 22 & NS \\
\hline $\mathrm{Nb}$. & 14 & 97 & 92 & 19 & 30 & 25 & 23 & 22 & & \\
\hline $\begin{array}{l}\% \\
\text { theo. }\end{array}$ & 4 & 30 & 29 & 6 & 9 & 8 & 7 & 7 & & \\
\hline
\end{tabular}

Tableau III. Précocité et réussite de la levée : $n$ : Nombre de plantes à levée précoce sur un segment; $N$ : Nombre total de plantes levées sur un segment; (..) : Nombre de segments de la catégorie; TH : Effectif théorique; OBS : Effectif observé.

\begin{tabular}{|c|c|c|c|c|c|c|c|c|c|c|c|}
\hline$N$ & $n$ & 8 & 7 & 6 & 5 & 4 & 3 & 2 & 1 & 0 & $\chi^{2}$ \\
\hline 8 & $\mathrm{TH}$ & & & 4,0 & 3,7 & 2,2 & & & & & \\
\hline (14) & OBS & 0 & 2 & 2 & 2 & 3 & 1 & 1 & 2 & 1 & THS \\
\hline 7 & $\mathrm{TH}$ & - & 1,8 & 5,8 & 8,1 & 6,3 & 3,0 & & 1,0 & - & \\
\hline (26) & OBS & - & 9 & 6 & 6 & 1 & 0 & 0 & 0 & 4 & THS \\
\hline 6 & $\mathrm{TH}$ & - & - & 1,2 & 3,4 & 3,9 & 2,4 & 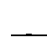 & 1,0 & & \\
\hline (12) & OBS & - & - & 5 & 5 & 1 & 0 & 1 & 0 & 0 & HS \\
\hline
\end{tabular}

Cette dernière a été calculée en considérant que la répartition fréquentielle du nombre de pieds précoces $n$ sur un segment de densité $\mathrm{N}$ suit une loi binomiale de puissance $N$, où $p$ et $q$ sont les proportions respectives de précoces et de tardifs. On constate que le calcul sous-estime les fréquences :

- des segments à dominante tardive, si $N=8$;

- des deux types, si $\mathrm{N}=7$.

Les manques à la levée correspondent donc préférentiellement aux segments à levée précoce.

Par conséquent, l'association spatiale privilégiée des précoces d'un côté, des tardifs de l'autre, a dû limiter l'occurrence d'une domination des seconds par les premiers. Toutefois une confusion d'effet pourrait se produire du fait de l'implantation moins dense des précoces.

\section{La croissance et la production finale}

La croissance présente les caractéristiques habituelles, de la levée à la floraison:
- une phase post-levée à vitesse de croissance d'abord en augmentation puis sensiblement constante jusqu'à la date 8 ;

- ensuite le taux de croissance se stabilise de la date 9 à la date 12;

- on observe enfin une phase à vitesse de croissance à peu près constante (dates 13 à 15) jusqu'à la floraison.

Pendant la première phase (Tableau IVa), le classement des moyennes de poids par groupe de levée est rigoureusement celui des dates de levée. A âge égal, apparaît une dépression progressive du poids selon la tardivité de levée, avec une certaine proximité de 1 et 2 d'une part, et des groupes plus tardifs d'autre part (Fig. 2).

Aux dates 9 et 10 (Tableau IVb), la corrélation Poids/Date de levée se retrouve; par la suite, le groupe le plus précoce et les 2 plus tardifs continuent d'être bien différenciés, et le groupe 2 reste, en général, supérieur aux suivants; pour les groupes intermédiaires ( 3 à 7 ), le classement est plus variable. 
Tableau IVa et IVb. Moyennes des estimations de poids sec individuel par groupe de levée, aux différentes dates de contrôle.

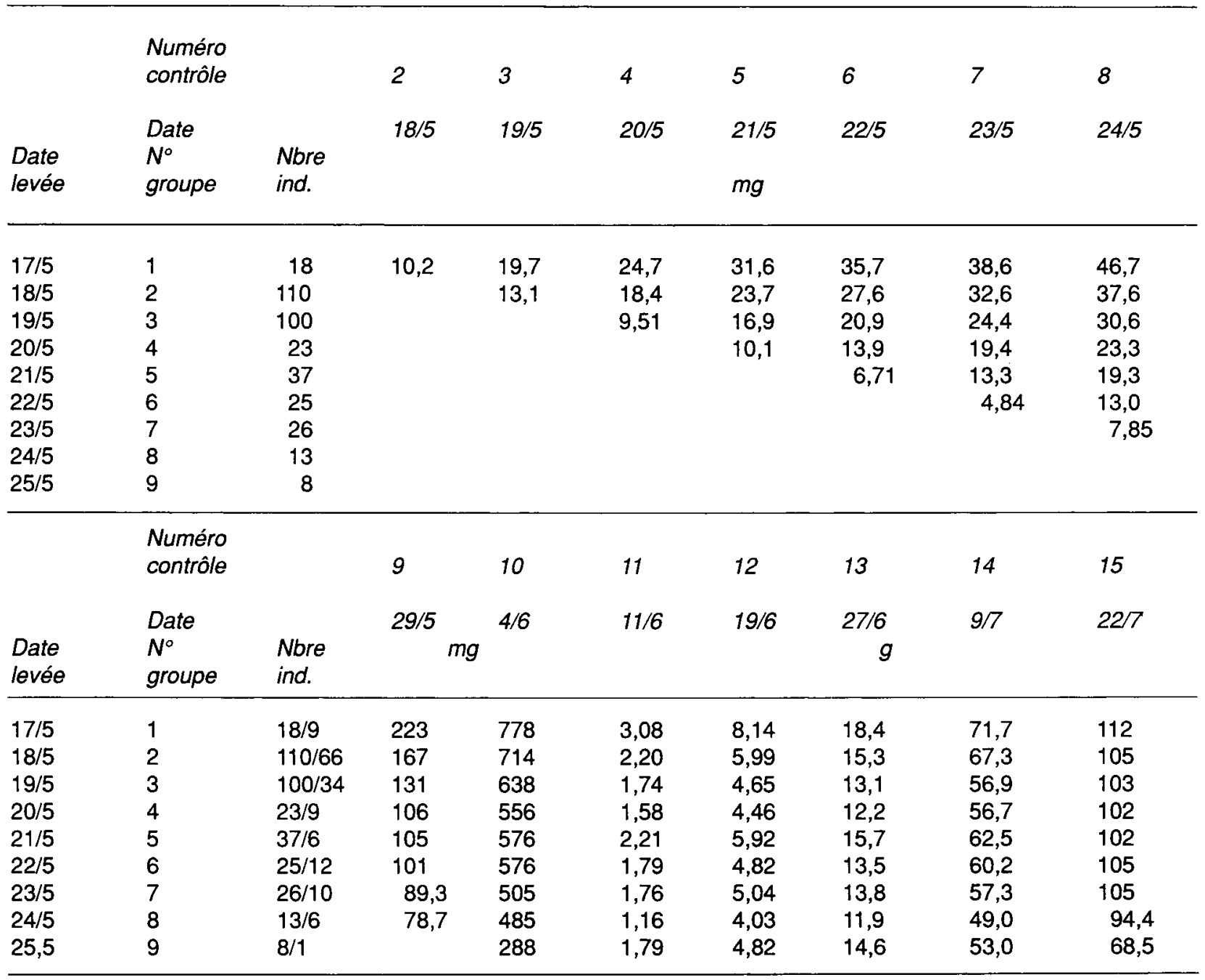

Tableau IVc. Moyennes des mesures à maturité par groupe de levée :

V : Matière sèche végétative (stér : plantes stériles comprises); NR : Nombre de rangs NG/NR : Nombre de grains par rang; P1000G : Poids de 1000 grains; PG : Poids de grain.

Maturité

\begin{tabular}{|c|c|c|c|c|c|c|c|c|c|}
\hline $\begin{array}{l}\text { Date } \\
\text { levée }\end{array}$ & $\begin{array}{l}N^{\circ} \\
\text { groupe }\end{array}$ & $\begin{array}{l}\text { Nbre } \\
\text { ind. }\end{array}$ & $\begin{array}{l}V \\
\text { Stér. } \\
g\end{array}$ & $\begin{array}{l}\% \\
\text { Stér. }\end{array}$ & $\begin{array}{l}V \\
g\end{array}$ & $N R$ & $N G / N R$ & $\begin{array}{l}P 1000 G \\
g\end{array}$ & $\begin{array}{l}P G \\
g\end{array}$ \\
\hline $17 / 5$ & 1 & 18 & 99,3 & 0 & 99,3 & 12,1 & 28,9 & 304 & 106 \\
\hline $18 / 5$ & 2 & 110 & 96,5 & 0 & 96,5 & 11,2 & 28,9 & 306 & 98,7 \\
\hline $19 / 5$ & 3 & 100 & 89,7 & 4 & 91,5 & 11,1 & 29,1 & 304 & 98,4 \\
\hline $20 / 5$ & 4 & 23 & 86,1 & 2 & 92,3 & 11,0 & 28,6 & 296 & 93,7 \\
\hline $21 / 5$ & 5 & 37 & 82,6 & 4 & 88,7 & 11,1 & 29,5 & 305 & 99 \\
\hline $22 / 5$ & 6 & 25 & 91,6 & 0 & 91,6 & 11,6 & 29,6 & 300 & 103 \\
\hline $23 / 5$ & 7 & 26 & 89,7 & 1 & 91,2 & 11,2 & 28,4 & 295 & 93,3 \\
\hline $24 / 5$ & 8 & 13 & 74,9 & 2 & 86,4 & 11,1 & 27,8 & 299 & 90,4 \\
\hline $25 / 5$ & 9 & 8 & 47,3 & 3 & 63,1 & 11,4 & 25,6 & 276 & 83,1 \\
\hline \multirow{2}{*}{\multicolumn{3}{|c|}{$\begin{array}{l}\text { niveau de significat. } \\
\text { écart-type erreur }\end{array}$}} & $\mathrm{HS}$ & & HS & NS & NS & NS & $S$ \\
\hline & & & & & 18,7 & 1,39 & 3,88 & 31,1 & 16,7 \\
\hline
\end{tabular}




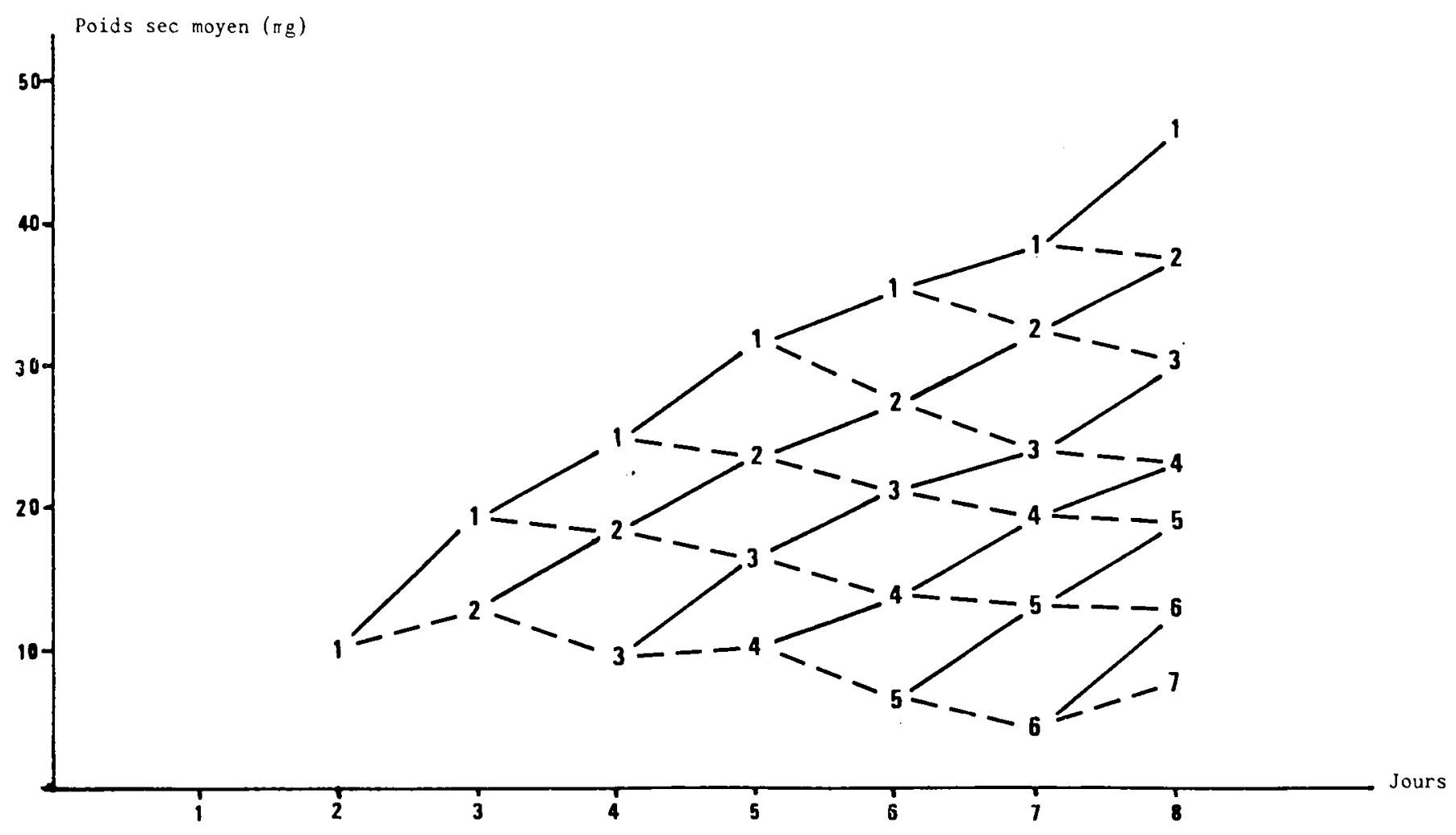

Fig. 2. Croissance après la levée. Groupes de levée; - - Etat de croissance de plantes de même âge calendaire; Courbe de croissance d'un groupe de levée.

Les résultats à la récolte (Tableau IVc) sont davantage regroupés pour le grain que pour l'appareil végétatif. Dans la mesure où l'on atteint le maximum variétal de production de grain, on se situe aussi dans la zone d'abaissement de l'harvest index décrit par Navarro Garza (1984). Les écarts les plus sensibles sont le fait des extrêmes :

- bien que l'analyse de la variance ne permette pas de différenciation significative pour cette composante, le groupe 1 montre une supériorité du nombre de rangs;

- le groupe 9 présente des dépressions de poids végétatif, de nombre et de poids unitaire de grain.

\section{La variabilité interne des groupes de levée}

$\mathrm{Si}$ les différences entre groupes initialement observées s'atténuent pour partie, l'hétérogénéité intragroupe est toujours élevée. La procédure d'évaluation du poids en limite l'analyse aux groupes de levée à fort effectif, c'est-à-dire 2 et 3. A la date 9 , on a trié entre pieds "légers" et "lourds" au sein de ces 2 groupes, en ména- geant une classe vide entre les 2 types; on constate que la séparation ainsi créée se perpétue aux dates ultérieures (Tableau $\mathrm{V}$ ) et, pour le poids végétatif, à la récolte. De plus, les différences entre ces sous-populations sont de même ordre que celles observées sur l'ensemble de la gamme de levées.

\section{Discussion}

La variabilité des productions individuelles à la récolte n'est explicitée qu'en partie par la variable date de levée. Sans le choix délibéré de réduire la compétition, habituelle en conditions normales de culture, cette variable aurait probablement procuré un pouvoir explicatif plus élevé. Jugée très tôt à une date donnée, la structure du peuplement a été marquée fortement par la différenciation des groupes de levée. En outre, à âge égal et juste après levée, on voit une certaine dépression des tardifs (Fig. 2). Mais une différenciation secondaire s'est surajoutée ultérieurement, modifiant la structure initiale. Ce sont ces différents points que nous allons chercher à expliciter. 
Tableau V. Moyenne des estimations de poids sec et des mesures à maturité pour les plantes des groupes de levée $n^{\circ} 2$ d'une part, et $n^{\circ} 3$ d'autre part, triées en légères et lourdes à l'issue de la phase hétérotrophe.

\begin{tabular}{|c|c|c|c|c|c|c|c|c|}
\hline $\begin{array}{l}\text { Numéro } \\
\text { de } \\
\text { contrôle }\end{array}$ & Date & Unité & $\begin{array}{l}\text { Levée le } \\
\text { faible } \\
\text { croiss. } \\
\text { hétéro. } \\
n=40\end{array}$ & $\begin{array}{l}\text { mai } \\
\text { forte } \\
\text { croiss. } \\
\text { hétéro. } \\
n=45\end{array}$ & $\begin{array}{l}\text { niveau } \\
\text { de } \\
\text { signif. }\end{array}$ & $\begin{array}{l}\text { Levée le } \\
\text { faible } \\
\text { croiss. } \\
\text { hétéro. } \\
n=39\end{array}$ & $\begin{array}{l}\text { mai } \\
\text { forte } \\
\text { croiss. } \\
\text { hétéro. } \\
n=42\end{array}$ & $\begin{array}{l}\text { niveau } \\
\text { de } \\
\text { signif. }\end{array}$ \\
\hline 9 & 29 & $\mathrm{mg}$ & ind & ind & & ind & ind & \\
\hline & mai & & $<150$ & $>170$ & & $<125$ & $>135$ & \\
\hline 10 & $\begin{array}{l}4 \\
\text { juin }\end{array}$ & - & 574 & 835 & & 489 & 779 & \\
\hline 11 & $\begin{array}{l}11 \\
\text { juin }\end{array}$ & $\mathrm{g}$ & 1,45 & 2,59 & & 1,13 & 2,31 & \\
\hline 12 & $\begin{array}{l}19 \\
\text { juin }\end{array}$ & - & 4,45 & 6,93 & & 3,51 & 5,74 & \\
\hline 13 & $\begin{array}{l}27 \\
\text { juin }\end{array}$ & - & 12,2 & 17,1 & & 10,5 & 15,4 & \\
\hline 14 & $\begin{array}{l}9 \\
\text { juillet }\end{array}$ & - & 58,2 & 72,8 & & 47,6 & 66,1 & \\
\hline 15 & $\begin{array}{l}22 \\
\text { juillet }\end{array}$ & - & 96,7 & 111 & HS & 93,5 & 112 & \\
\hline V & maturité & - & 88,3 & 101 & HS & 86,4 & 100 & HS \\
\hline NR & - & & 11,1 & 11,5 & NS & 11 & 11,3 & NS \\
\hline $\mathrm{NG} / \mathrm{NR}$ & - & & 28,5 & 29 & NS & 28,4 & 29,3 & NS \\
\hline P1000G & - & - & 300 & 306 & NS & & & \\
\hline$P G$ & - & - & 94,9 & 103 & $\mathrm{~s}$ & 95,3 & 101 & NS \\
\hline
\end{tabular}

\section{Etude de la levée}

L'existence d'une différenciation de la date de levée est habituellement reconnue, mettant en cause la variété des situations écologiques des semences et leur propriété germinative. L'association préférentielle des manques à des segments à levée précoce pose cependant question. Elle pourrait s'expliquer de la façon suivante : la précocité refléterait une faible profondeur de semis et le dessèchement du lit de semence à partir de la surface empêcherait l'imbibition convenable des graines trop faiblement en contact avec les agrégats de terre.

Les pieds disparus en cours de végétation, ou à très faible production (moins de $30 \mathrm{~g}$ de poids végétatif et moins de $30 \mathrm{~g}$ de grain) sont peu nombreux (11); le rôle des déprédateurs dans ces accidents doit être faible car ceux-ci ne se produisent qu'en groupes 4 et 5 et dans les groupes très tardifs ( 8 à 10$)$ où ils représentent $27 \%$ de l'effectif; leurs voisins sont à $89 \%$ des pieds précoces. On serait tenté d'y voir un effet de compétition si leur disparition, ou l'acquisition du caractère chétif, n'était très précoce (au plus tard à la date 10). Le stade végétatif des autres plantes, alors de 4 à 6 feuilles, fait plutôt penser à une conséquence du passage à l'autotrophie. Cette localisation, jointe à celle des manques à la levée, conduit à proposer une partition a posteriori des pieds tardifs, en fonction de la précocité de leurs voisins :

- en zone tardive, où le comportement est normal, la cause de tardivité ne pèserait pas sur le fonctionnement : on peut penser à une imbibition lente, telle que l'ont analysée Boiffin et al. (1983), à une profondeur de semis supérieure, ou à l'existence d'obstacles devant être contournés entre la semence et la surface;

- en zone précoce, la cause de tardivité serait pénalisante totalement (non levée) ou partiellement (pieds chétifs ou à disparition ultérieure); dans ce dernier cas, elle pourrait être d'origine génétique.

\section{Différenciation des courbes de croissance selon les groupes de levée}

\section{Croissance précoce}

En l'absence de contrôle de la température du sol, on a utilisé l'âge calendaire comme mesure du temps dans la Figure 2. Or, la température de l'air diminuant au cours de la levée, il doit en être de même de celle du lit de semence; il s'ensuit que l'âge physiologique des dernières levées est surestimé, donc leur vitesse de croissance surévaluée, ce qui explique leur retard relatif signalé plus haut. 
Evolution de la variabilité entre groupes de levée

Compte tenu de la très forte corrélation entre les 2 variables, on a considéré qu'à la date 9 les différences de poids provenaient, pour l'essentiel, des différences d'âge. En outre, la majorité des plantes ayant atteint le stade 4 feuilles, leur fonctionnement est autotrophe ce qui autorise à analyser leur croissance selon le modèle exponentiel. On en a calculé les paramètres en effectuant la régression linéaire du logarithme du poids sur la somme des températures aériennes, ce qui donne:

$$
P=21,46 \exp (0,036 t)
$$

( $P$ en $\mathrm{mg}$, $t$ en $\mathrm{d}^{\circ} \mathrm{j}$, base 10 ).

Cette valeur du taux de croissance, voisine de celle évaluée par Fleury et al. (1986), nous autorise à utiliser l'équation ci-dessus pour estimer les poids secs moyens de chaque groupe de levée aux dates ultérieures. Sur la Figure 3 , les valeurs calculées sont mises en regard des valeurs observées : les groupes précoces se distinguent par une surestimation systématique quoique faible. La réduction de la variabilité entre groupes pourrait donc être attribuée à un ralentissement relatif des précoces par rapport aux tardifs. Les conditions de croissance des précoces seraient donc moins bonnes après l'autotrophie.

\section{Evolution de la variabilité intragroupe}

Le même modèle de croissance a été appliqué de façon analogue aux 2 sous-populations des groupes 2 et 3 . Les équations sont les suivantes:

$$
\begin{aligned}
\text { - Groupe } 2: \text { Légers } & P=17,11 \exp (0,0335 t) \\
\text { Lourds } & P=29,37 \exp (0,0330 t) \\
\text { - Groupe } 3: \text { Légers } & P=18,05 \exp (0,0330 t) \\
\text { Lourds } & P=28,59 \exp (0,0338 t)
\end{aligned}
$$

La variabilité constatée relève donc surtout de celle du poids à l'origine, qui distingue de façon uniforme "légers" et “lourds" dans les 2 groupes.

Les mesures quotidiennes étaient effectuées approximativement à la même heure, aussi certaines plantes répertoriées dans le même groupe peuvent, à la limite, avoir jusqu'à près de $24 \mathrm{~h}$ de

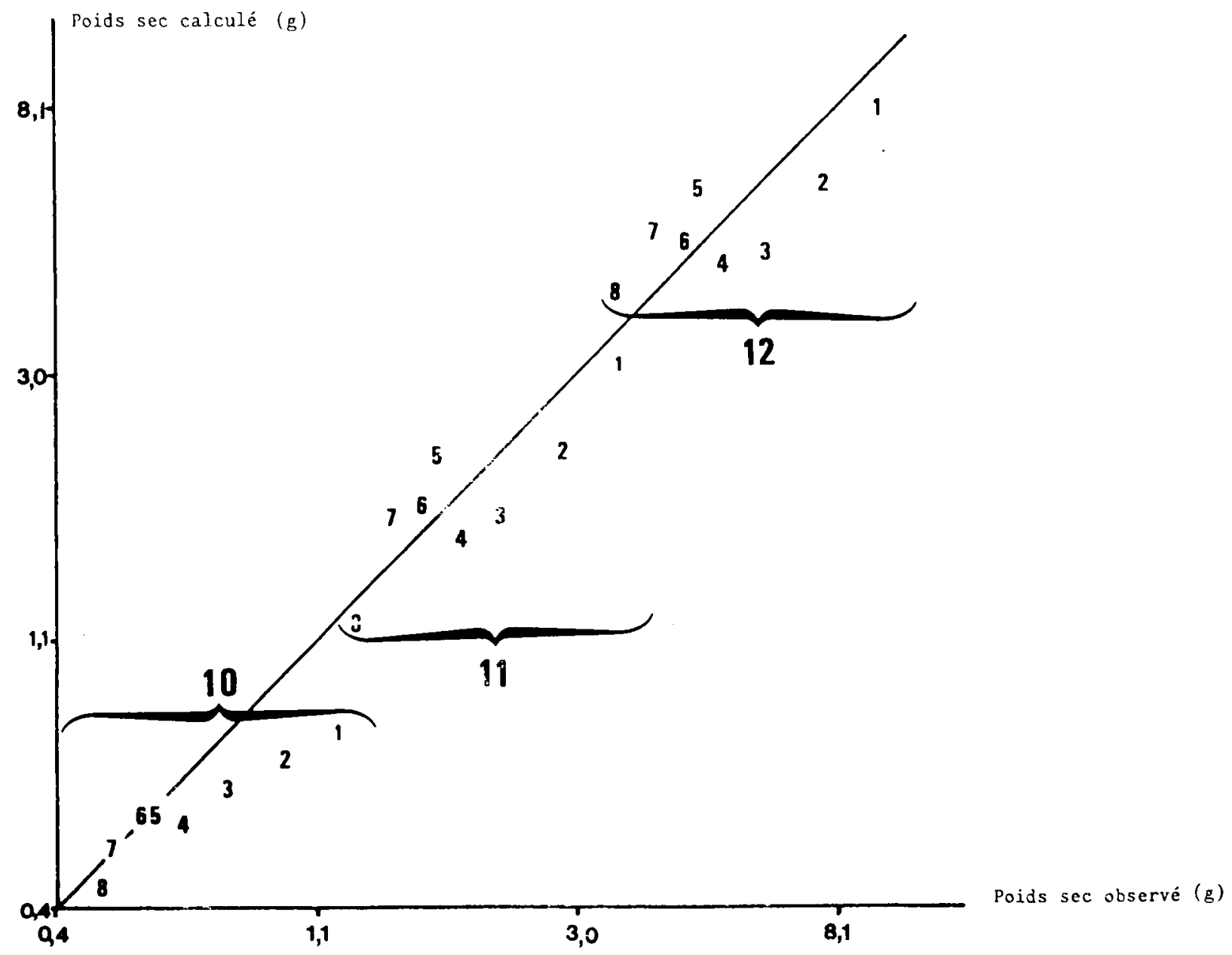

Fig. 3. Poids observés et calculés d'après les résultats du prélèvement 9.10 Numéro du prélèvement; 2 Groupe de levée. 
différence d'âge, et d'autres répertoriées dans 2 groupes successifs, n'en avoir presque aucune; toutefois la différence observée est trop importante pour ne relever que d'un décalage d'heure de levée au cours de la même journée; elle pourrait traduire la variabilité propre du lot de semence concernant surtout l'importance des réserves de la graine, la vitesse et l'intégralité de leur utilisation.

\section{Conclusion}

Lors des stades précoces du maïs, la variabilité interne du peuplement est directement inféodée aux dates de levée et aux états initiaux de croissance. Dans la suite du cycle, la différenciation ainsi créée s'amoindrit relativement et ne continue d'être observable que lorsque l'écart en sommes de températures séparant les levées est suffisamment important, et le nombre de plantes, contrôlées dans chaque groupe, suffisamment élevé.

Notre protocole expérimental visait à offrir à chaque individu les possibilités optimales d'extériorisation de ses potentialités, par la limitation de la compétition et la récolte à la maturité physiologique. La conséquence de ce choix est qu'effectivement chaque catégorie de levée ou d'état initial atteint ou approche les maxima variétaux. Dans des conditions de culture plus habituelles - c'est-à-dire à densité élevée et avec une récolte antérieure à la fin physiologique du cycle de la plante - on aurait sans doute obtenu une différenciation finale plus marquée.

Bien que facteur favorable, la précocité de levée est spatialement associée à des manques; au cours du cycle de la plante, des difficultés de croissance lui sont liées. Ces observations rappellent qu'on ne peut considérer la structure du peuplement comme définitivement déterminée sitôt son effectif établi, du fait de l'expression hiérarchisée des facteurs et conditions de différente nature, intervenant au cours des phases successives. En d'autres termes, lorsqu'on étudie la structure du peuplement à un moment donné, il faut y voir conjointement :

- d'une part, l'héritage, bien net au moment du passage à l'autotrophie, des différences existant entre caryopses pour la taille, la qualité semencière et la situation écologique. En ce qui concerne le dernier point, insistons sur la nécessité, lors d'études ultérieures, de décrire précisément l'environnement immédiat des semences : en effet, la répartition des agrégats et des vides autour de la graine contrôle largement les conditions hydriques, thermiques et d'oxygénation de celleci et pourraient être responsables d'une part importante de la variabilité observée;
- d'autre part, l'effet de la variabilité du milieu se manifestant sur les plantes tout au long de leur croissance.

Pour terminer, il faut souligner la nécessité de faire appel à la température - celle du sol serait préférable - dans l'analyse des comportements précoces.

\section{Références}

Abd el Rahman N. \& Bourdu R. (1986) Effet de la taille et de la forme des grains sur quelques caractéristiques du développement du maiis au stade jeune. Agronomie 6, 181-186

Abdul Hamid I. (1986) Influence de la qualité semencière sur la germination, la croissance hétérotrophe et le devenir des plantules de maïs. Thèse Doct. Univ. Rennes

Belfqih A. \& Charbey V. (1984) Caractérisation de la structure de peuplement de la betterave sucrière. Mémoire de DAA. Chaire d'Agronomie, INA-PG, Paris

Boiffin J., Bruckler L. \& Aubry C. (1983) Rôle des propriétés physiques du lit de semences sur l'imbibition et la germination. III - Valeur prévisionnelle d'un modèle d'imbibition au champ et caractérisation des lits de semences. Agronomie 3, 291-302

Duncan W.G., Hammond L.C., Robertson W.K. \& Lundy H.W. (1972) Effects of environmental stress and planting accuracy on plant-to-plant variability in corn (Zea mays L.). Soil Crop Sci. Soc. Florida, Proc. 32, 14

Edmeades G.D. \& Daynard T.B. (1979) The development of a plant-to-plant variability in maize at different planting densities. Can. J. Plant. Sci. 59, 561-576

Fleury A. \& Caneill J. (1983) Croissance et développement de la betterave sucrière; analyse de l'hétérogénéité du peuplement. Séminaire du Département d'Agronomie, Chambon du Lac, INRA, Paris, 1113/10/83

Fleury A., Bertrand M. \& Ronsin T. (1986) Essai d'analyse climatique de la croissance précoce du maïs. C.R. Acad. Agric. Fr. 72, 501-602 (Séance du 18/06/86)

Glenn F.B. \& Daynard T.B. (1974) Effects of genotype, planting pattern and plant density on plant-to-plant variability and grain yield of corn. Can J. Plant. Sci. 54, $323-330$

Hernandez A. (1987) Compte rendu des essais "Croissance et développement du maïs», RNED Céréales

Maillet (1987) Contribution à l'étude de la croissance juvénile de la betterave sucrière. Mémoire de DEA, Chaire d'Agronomie, INA-PG, Paris

Navarro Garza H. (1984) L'analyse des composantes du rendement du maïs. Application à l'étude de la variabilité du rendement dans une petite région. Thèse DDI, INA-PG

Pendleton J.W. \& Seif R.D. (1962) Role of height in corn competition. Crop Sci. 2, 154-156

Pommel B. (1979) Comparaison de l'utilisation par le maïs du phosphore des boues résiduaires ou du phosphate monocalcique pour différents niveaux de zinc et de fer dans le substrat. Ann. Agron. 30, 443-453 